Proceedings of the Edinburgh Mathematical Society (2005) 48, 125-142 (C)

DOI:10.1017/S0013091503001081 Printed in the United Kingdom

\title{
WHEN IS A UNIT LOOP $f$-UNITARY?
}

\author{
EDGAR G. GOODAIRE ${ }^{1}$ AND CÉSAR POLCINO MILIES ${ }^{2}$ \\ ${ }^{1}$ Memorial University of Newfoundland, St. John's, \\ NL A1C 5S7, Canada (edgar@math.mun.ca) \\ ${ }^{2}$ Instituto de Matemática e Estatística, Universidade de São Paulo, \\ Caixa Postal 66.281, CEP 05311-970, São Paulo SP, \\ Brazil (polcino@ime.usp.br)
}

(Received 9 December 2003)

\begin{abstract}
Let $L$ be an RA loop, that is, a loop whose loop ring in any characteristic is an alternative, but not associative, ring. Let $f: L \rightarrow\{ \pm 1\}$ be a homomorphism and, for $\alpha=\sum \alpha_{\ell} \ell \in \mathbb{Z} L$, define $\alpha^{f}=\sum f(\ell) \alpha_{\ell} \ell^{-1}$. Call $\alpha f$-unitary if $\alpha^{f}=\alpha^{-1}$ or $\alpha^{f}=-\alpha^{-1}$. In this paper, we identify the RA loops $L$ with the property that all units in $\mathbb{Z} L$ are $f$-unitary. Along the way, we extend a famous theorem of Higman to a case still undecided in group rings.
\end{abstract}

Keywords: loop ring; alternative ring; Moufang loop

2000 Mathematics subject classification: Primary 20N05

Secondary 17D05; 16S34; 16U60

\section{Introduction}

A loop ring is an algebraic object $R L$, constructed in the same way as a group ring, but in which the underlying loop $L$ is not necessarily associative. This paper is concerned with loop rings which are alternative, but not associative. Loops which give rise to such loop rings (over commutative associative rings $R$ of any characteristic) are called $R A$ (ring alternative) loops. The best reference for information about RA loops and their loop rings is the monograph [15], though we record here some properties of particular relevance to this paper.

RA loops are Moufang and hence dissociative; that is, the subloop generated by two elements is always associative (so parentheses are never needed to indicate order of multiplication in monomials). We use implicitly throughout this paper the fact that if two elements of an RA loop commute, then they associate with every third element [15, Theorem IV.1.1 and Corollary IV.1.3]. An RA loop $L$ has the so-called $L C$ property; namely, elements $x, y \in L$ commute if and only if $x$ or $y$ or $x y$ is central [15, $\S$ IV.2] (see also [6]). An RA loop $L$ possesses a special element (which we always label $s$ ) that is both a unique non-identity commutator and a unique non-identity associator; that is, if $a, b \in L$ do not commute, then $b a=s a b$ and, if $a, b, c \in L$ do not associate, then $(a b) c=[a(b c)] s$. (It is 
easy to see that $s$ is necessarily central and of order 2.) Letting $(a, b)$ denote the commutator of $a$ and $b$, and $(a, b, c)$ the associator of $a, b$ and $c$, we then have that for any $a, b, c \in L$,

$$
(a, b)=1 \text { or } s \quad \text { and } \quad(a, b, c)=1 \text { or } s .
$$

In $L$, the map

$$
\ell \mapsto \ell^{*}= \begin{cases}\ell & \text { if } \ell \text { is central, } \\ s \ell & \text { if } \ell \text { is not central, }\end{cases}
$$

for $\ell \in L$ extends (by linearity) to an involution (that is, an antiautomorphism of period two) of the alternative ring $R L$.

Finally, we note that an RA loop $L$ is generated by its centre and any three elements $x, y, u \in L$ that do not associate. Letting $G$ be the group generated by $x$ and $y$, the loop $L$ is completely determined by $G, *$ and $u^{2}$ (see [15, $\S$ II.5.2 and Theorem IV.3.1]), so we use the notation $L=M\left(G, *, u^{2}\right)$.

Let $f: L \rightarrow\{ \pm 1\}$ be a homomorphism and, for $\alpha=\sum \alpha_{\ell} \ell \in R L$, define $\alpha^{f}=$ $\sum f(\ell) \alpha_{\ell} \ell^{-1}$. We say that $\alpha$ is $f$-unitary if $\alpha^{f}=\alpha^{-1}$ or $\alpha^{f}=-\alpha^{-1}$. It is easy to verify that $\alpha \mapsto \alpha^{f}$ is an antiautomorphism of the loop ring and that the set $\mathcal{U}_{f}(R L)$ of $f$-unitary units of $R L$ is a subloop of the full loop $\mathcal{U}(R L)$ of units.

The concept of an $f$-unitary unit in a group ring $R G$ was introduced by Bovdi [1]. In the special case in which $f(g)=1$ for all $g \in G, \alpha^{f}$ is generally denoted $\alpha^{*}$ and a unit is called simply unitary if $\alpha^{*}=\alpha^{-1}$. This situation has received quite a bit of attention recently. Bovdi and Kovacs determined when $\mathcal{U}_{*}(K G)$ ( $K$ a field) is normal in $\mathcal{U}(K G)[4]$. Bovdi and Erdei have considered the possibility that a group may have a normal complement in the unitary group $\mathcal{U}_{*}(\mathbb{Z} L)[\mathbf{3}]$. Gonçalves and Passman have studied groups $G$ whose unitary subgroup in $K G$ does not contain a free group [9], and Giambruno and Polcino Milies determined conditions for this subgroup to satisfy a group identity [8].

The purpose of this paper is to determine precisely which RA loops $L$ have the property that every unit in the integral loop ring $\mathbb{Z} L$ is $f$-unitary (for some $f$ ). Equivalently, when is it the case that $\mathcal{U}_{f}(\mathbb{Z} L)=\mathcal{U}(\mathbb{Z} L)$ ? This problem has been studied for group rings by Bovdi and Sehgal $[\mathbf{1}, \mathbf{2}]$.

In the next two sections, we give instances of where all units are $f$-unitary and then, in $\S 4$, we show that our list is complete. Interestingly, our investigations led to an extension to arbitrary RA loops of a theorem of Higman, a generalization still undecided in group rings (see Theorem 2.3).

\section{A generalized theorem of Higman in the alternative case}

An element $\ell$ in a group (or loop) $L$ is torsion if $\ell^{n}=1$ for some $n \in \mathbb{N}$. If $L$ is an RA loop, the set of torsion elements forms a subloop of $L$, in fact, a subloop that is locally finite and normal [15, Theorem VIII.4.1]. One of the earliest, most-fundamental (and oft-quoted) results in the theory of group rings is due to Graham Higman, who determined when all the units in an integral group ring $\mathbb{Z} G$ (with $G$ torsion) are trivial in the sense that $\mathcal{U}(\mathbb{Z} G)= \pm G=\{ \pm g \mid g \in G\}$. 
Theorem 2.1 (see [18]). If $G$ is a torsion group, then all units in $\mathbb{Z} G$ are trivial if and only if $G$ is

(1) an abelian group of exponent dividing 4 or 6 , or

(2) a Hamiltonian 2-group.

This theorem has been generalized to loops whose loop rings are alternative, but not necessarily associative (see [12] but also [15, Theorem VIII.3.2] for an elegant modern proof).

Theorem 2.2. Let $L$ be a torsion $R A$ loop. Then all units in $\mathbb{Z} L$ are trivial if and only if $L$ is a Hamiltonian Moufang 2-loop.

Recently, we have found another theorem characterizing RA loops with trivial unit loops without any restriction on the loop. Recall that the augmentation map on a loop ring $R L$ is the homomorphism $\epsilon: R L \rightarrow R$ defined by $\epsilon(\alpha)=\sum \alpha_{\ell}$ for $\alpha=\sum \alpha_{\ell} \ell \in R L$. The scalar $\epsilon(\alpha)$ is called the augmentation of $\alpha$.

Theorem 2.3. Let $L$ be an $R A$ loop with torsion subloop $T$. Then all units of $\mathbb{Z} L$ are trivial if and only every subloop of $T$ is normal in $L$, and $T$ is an abelian group of exponent dividing 4 or 6 or a Hamiltonian Moufang 2-loop.

Proof. Our argument, and many others in this paper, uses the fact that in an RA loop (unlike in Moufang loops in general), the test for normality of a subloop is the same as it is for groups [15, Corollary IV.1.11] (see also [7, Corollaries 2.4 and 2.11]).

Assume that all units of $\mathbb{Z} L$ are trivial. In particular then, $\mathcal{U}(\mathbb{Z} T)= \pm T$, which is a torsion group or a torsion $R A$ loop. Thus, by Theorems 2.1 and 2.2, $T$ is an abelian group of exponent dividing 4 or 6 or a Hamiltonian Moufang (possibly associative) 2-loop. Next, let $t \in T, x \in L$ and suppose that $x^{-1} t x \notin\langle t\rangle$. Let $\hat{t}=1+t+t^{2}+\cdots+t^{n-1}$, where $n$ is the order of $t$. The element $(1-t) x \hat{t}$ has square 0 , so $1+(1-t) x \hat{t} \in \mathbb{Z} L$ is a unit and is not trivial [15, Lemma VIII.2.2]. This contradiction shows that every subloop of $T$ is normal in $L$.

Conversely, assume that every subloop of $T$ is normal in $L$ and that $T$ is an abelian group of exponent dividing 4 or 6 or a Hamiltonian Moufang 2-loop. In any event, $T$ has an exponent dividing 4 or 6 . In an RA loop $L$, squares are central, so elements of odd order are also central [15, Theorem IV.1.8] (see also [10]). If $t \in L$ has order 2, then $t$ too must be central since, for any $x \in L, x^{-1} t x \in\langle t\rangle=\{1, t\}$. There are no non-central elements $t$ of order 6 , since $x^{-1} t x=s t=t^{5}$ implies $t^{4}=s$ and $t^{8}=1$, a contradiction. It follows that any non-central element in $T$ must have order 4 . If $\xi$ is a primitive fourth root of unity, the map $\sigma: \xi \mapsto \xi^{(4 / 2)+1}$ is in the Galois group of $\mathbb{Q}(\xi) / \mathbb{Q}$, so every idempotent of $\mathbb{Q} T$ is central in $\mathbb{Q} L[\mathbf{1 5}$, Theorem XIII.1.10] (see also [14, Theorem 3.3]).

Now let $\mu \in \mathcal{U}(\mathbb{Z} L)$. Replacing $L$ by the subloop generated by a finite set containing the support of $\mu$ and three elements which do not associate, we may assume that $L$ is finitely generated. By Corollary XIII.2.2 of $[\mathbf{1 5}], \mathbb{Q} L$ contains no (non-zero) nilpotent elements, so the same applies to $\mathbb{Q} T$, which is therefore the direct sum of division rings. Now Corollary XII.1.2 of [15] (see also [13, Lemma 2.3]) says that $\mathcal{U}(\mathbb{Z} L)=L \cdot \mathcal{U}(\mathbb{Z} T)$. Since $\mathcal{U}(\mathbb{Z} T)= \pm T$ by Theorem 2.2 , the result follows. 
Corollary 2.4. Let $L$ be an RA loop with torsion subloop $T$. If every subloop of $T$ is normal in $L$, and $T$ is either an abelian group of exponent dividing 4 or 6 or a Hamiltonian Moufang 2-loop, then every unit in $\mathbb{Z} L$ is $f$-unitary (for any homomorphism $f: L \mapsto\{ \pm 1\})$.

Proof. The hypothesis and Theorem 2.3 imply that if $\mu \in \mathbb{Z} L$ is a unit, then $\mu= \pm \ell$ for some $\ell \in L$. For any $f: L \mapsto\{ \pm 1\}, \mu^{f}= \pm \ell^{-1}= \pm \mu^{-1}$, so $\mu$ is $f$-unitary.

\section{Further examples}

In this section, we give further instances of RA loops $L$ for which all units of $\mathbb{Z} L$ are $f$-unitary.

We begin with a useful lemma, first established for groups by Li [19, Theorem 2.1].

Lemma 3.1. Let $L_{0}$ be an $R A$ loop and let $f: L_{0} \rightarrow\{ \pm 1\}$ be a non-trivial homomorphism. Let $E$ be an abelian group of exponent two, let $L=L_{0} \times E$ and extend $f$ to $f_{1}: L \rightarrow\{ \pm 1\}$ by setting $f_{1}(E)=1$. If every unit of $\mathbb{Z} L_{0}$ is $f$-unitary, then every unit of $\mathbb{Z} L$ is $f_{1}$-unitary.

Proof. Let $\mu \in \mathcal{U}(\mathbb{Z} L)$. Then $\mu$ is a finite integral linear combination of terms of the form $\ell e, \ell \in L_{0}, e \in E$. Thinking of $E$ as a vector space over the field of two elements, each such $e$ is a finite linear combination of basis elements. It follows that $\mu$ is a linear combination of terms in a loop $L_{0} \times C_{2} \times C_{2} \times \cdots \times C_{2}$, so it suffices to establish the lemma for the case $L=L_{0} \times\langle c\rangle, c^{2}=1$.

Write $\mu=\mu_{1}+\mu_{2} c, \mu_{1}, \mu_{2} \in \mathbb{Z} L_{0}$. Let $\nu=\nu_{1}+\nu_{2} c=\mu^{-1}, \nu_{1}, \nu_{2} \in \mathbb{Z} L_{0}$. The equation $\mu \nu=1$ implies

$$
\mu_{1} \nu_{1}+\mu_{2} \nu_{2}=1
$$

and

$$
\mu_{1} \nu_{2}+\mu_{2} \nu_{1}=0
$$

so $\left(\mu_{1} \pm \mu_{2}\right)\left(\nu_{1} \pm \nu_{2}\right)=1$. Thus $\mu_{1}+\mu_{2}$ and $\mu_{1}-\mu_{2}$ are units in $\mathbb{Z} L_{0}$ and hence $f$-unitary. It follows that

$$
\left(\mu_{1}+\mu_{2}\right)^{f}= \pm\left(\mu_{1}+\mu_{2}\right)^{-1}= \pm\left(\nu_{1}+\nu_{2}\right)
$$

and

$$
\left(\mu_{1}-\mu_{2}\right)^{f}= \pm\left(\mu_{1}-\mu_{2}\right)^{-1}= \pm\left(\nu_{1}-\nu_{2}\right)
$$

In the case in which all the signs here are the same, we obtain $\mu_{1}^{f}= \pm \nu_{1}, \mu_{2}^{f}= \pm \nu_{2}$, so $\mu_{1}^{f}= \pm \nu_{1}$ and $\mu^{-1}=\nu_{1}+\nu_{2} c=\mu_{1}^{f}+\nu_{2}^{f} c= \pm \mu^{f_{1}}$. We can complete the proof, therefore, by showing that neither 'mixed case' can occur. Suppose, for example, that $\left(\mu_{1}+\mu_{2}\right)^{f}=\left(\mu_{1}+\mu_{2}\right)^{-1}$ but $\left(\mu_{1}-\mu_{2}\right)^{f}=-\left(\mu_{1}-\mu_{2}\right)^{-1}$. Thus

$$
\left(\mu_{1}+\mu_{2}\right)\left(\mu_{1}^{f}+\mu_{2}^{f}\right)=1=\left(\mu_{1}-\mu_{2}\right)\left(-\mu_{1}^{f}+\mu_{2}^{f}\right) .
$$


We obtain

$$
\begin{aligned}
\mu_{1} \mu_{1}^{f}+\mu_{1} \mu_{2}^{f}+\mu_{2} \mu_{1}^{f}+\mu_{m} u_{2}^{f} & =1, \\
-\mu_{1} \mu_{1}^{f}+\mu_{1} \mu_{2}^{f}+\mu_{2} \mu_{1}^{f}-\mu_{m} u_{2}^{f} & =1,
\end{aligned}
$$

and addition gives

$$
\mu_{1} \mu_{2}^{f}+\mu_{2} \mu_{1}^{f}=1 .
$$

Let $\mu_{1}=\sum_{\ell \in L_{0}} \alpha_{\ell} \ell, \alpha_{\ell} \in \mathbb{Z}$, and $\mu_{2}=\sum_{\ell \in L_{0}} \beta_{\ell} \ell, \beta_{\ell} \in \mathbb{Z}$. Then $\mu_{1}^{f}=\sum f(\ell) \alpha_{\ell} \ell^{-1}$, $\mu_{2}^{f}=\sum f(\ell) \beta_{\ell} \ell^{-1}$, and the coefficient of 1 on the left-hand side of (3.1) is a sum of terms of the form $f(\ell) \alpha_{\ell} \beta_{\ell}+f(\ell) \beta_{\ell} \alpha_{\ell}$, which is even, a contradiction. The proof of the other mixed case, $\left(\mu_{1}+\mu_{2}\right)^{f}=-\left(\mu_{1}+\mu_{2}\right)^{-1},\left(\mu_{1}-\mu_{2}\right)^{f}=+\left(\mu_{1}-\mu_{2}\right)^{-1}$, is similar.

In the next three theorems, we give specific examples of loops $L$ for which all units in $\mathbb{Z} L$ are unitary.

Theorem 3.2. Let $L=\mathcal{C} \times\langle b\rangle \times E$ be the direct product of the Cayley loop, $\mathcal{C}$, a cyclic group $\langle b\rangle$ of order 4 and a (possibly trivial) abelian group $E$ of exponent two. Let $A=\mathcal{C} \times\left\langle b^{2}\right\rangle \times E$. Then every unit in $\mathbb{Z} L$ is $f$-unitary, where $\operatorname{ker} f=A$.

Proof. Because of Lemma 3.1, we may assume that $E$ is trivial. Let $\mu$ be a unit of $\mathbb{Z} L$. Without loss of generality, the augmentation of $\mu$ is 1 . Now $\bar{\mu}$ is a unit in the integral loop ring of $L /\left\langle b^{2}\right\rangle$, which is a Hamiltonian 2-loop, so $\bar{\mu}=\bar{\ell}$ for some $\ell \in L$ (Theorem 2.2), hence $\mu=\ell+\left(1-b^{2}\right) \alpha$ for some $\alpha \in \mathbb{Z} L$. Similarly, $\bar{\mu}=\mathcal{U}\left(\mathbb{Z}\left[L / L^{\prime}\right]\right)$ is trivial, so $\bar{\ell}+\left(\overline{1}-\bar{b}^{2}\right) \bar{\alpha}=\bar{k}$ for some $k \in L$. Multiplying by $\overline{1}+\bar{b}^{2}$, we obtain

$$
\bar{\ell}\left(\overline{1}+\bar{b}^{2}\right)=\bar{k}\left(\overline{1}+\bar{b}^{2}\right),
$$

so $\bar{\ell}=\bar{k}$ or $\bar{\ell}=\bar{b}^{2} \bar{k}$. In the latter case, $\mu=b^{2} \ell+(1-s) \beta, \beta \in \mathbb{Z} L$ and, since $b^{2} \ell=$ $\ell-\left(1-b^{2}\right) \ell$,

$$
\mu=\ell+\left(1-b^{2}\right) \alpha=\ell+(1-s) \gamma,
$$

for some $\gamma \in \mathbb{Z} L$, so $\left(1-b^{2}\right) \alpha=(1-s) \gamma$. Write $\gamma=\gamma_{0}+\gamma_{1} b+\gamma_{2} b^{2}+\gamma_{3} b^{3}, \gamma_{i} \in \mathbb{Z C}$. In a similar way, write $\alpha=\alpha_{0}+\alpha_{1} b+\alpha_{2} b^{2}+\alpha_{3} b^{3}$, but note that

$$
\left(1-b^{2}\right) \alpha=\left(1-b^{2}\right)\left[\left(\alpha_{0}-\alpha_{2}\right)+\left(\alpha_{1}-\alpha_{3}\right) b\right] .
$$

Thus we may assume that $\alpha_{2}=\alpha_{3}=0$, hence that $\alpha=\alpha_{0}+\alpha_{1} b$. Consider the equation

$$
(1-s)\left(\gamma_{0}+\gamma_{1} b+\gamma_{2} b^{2}+\gamma_{3} b^{3}\right)=\left(1-b^{2}\right)\left(\alpha_{0}+\alpha_{1} b\right)=\alpha_{0}+\alpha_{1} b-\alpha_{0} b^{2}-\alpha_{1} b^{3} .
$$

Since the supports of $\alpha_{0}$ and $\alpha_{1} b$ are disjoint, and since the supports of $\gamma_{0}, \gamma_{1} b, \gamma_{2} b^{2}, \gamma_{3} b^{3}$ are also disjoint, it follows that $\alpha_{0}=(1-s) \gamma_{0}$ and $\alpha_{1}=(1-s) \gamma_{1}$, so $\alpha=(1-s)\left(\gamma_{0}+\gamma_{1} b\right)$ and $\mu=\ell+\left(1-b^{2}\right)(1-s) \gamma, \ell \in L$, and $\gamma=\gamma_{0}+\gamma_{1} b, \gamma_{0}, \gamma_{1} \in \mathbb{Z C}$. Thus

$$
\mu^{f}= \pm \ell^{-1}+\left(1-b^{2}\right)(1-s) \gamma^{f}
$$

and

$$
\mu \mu^{f}= \pm 1+\left(1-b^{2}\right)(1-s)\left[\ell \gamma^{f} \pm \gamma \ell^{-1}+4 \gamma \gamma^{f}\right]
$$


There are two cases to consider, according to whether $\ell \in \mathcal{C}$ or $\ell \in \mathcal{C} b$, but before we do so, it is important to observe that since $f(\ell)=1$ and

$$
\ell^{-1}= \begin{cases}\ell & \text { if } \ell \text { is central, } \\ s \ell & \text { if } \ell \text { is not central, }\end{cases}
$$

for all $\ell \in \mathcal{C}$, we have $\ell^{-1}=\ell^{*}$ (see (1.1)) and the restriction of the map $f$ to $\mathbb{Z C}$ is the canonical involution $\alpha \mapsto \alpha^{*}$ in an alternative loop ring.

Case 1. If $\ell \in \mathcal{C}$, then

$$
\mu \mu^{f}=+1+\left(1-b^{2}\right)(1-s)\left[\ell \gamma^{f}+\gamma \ell^{-1}+4 \gamma \gamma^{f}\right] .
$$

Remembering that $b$ is central, we have

$$
\begin{aligned}
\ell \gamma^{f}+\gamma \ell^{-1} & =\ell\left(\gamma_{0}^{*}-\gamma_{1}^{*} b^{-1}\right)+\left(\gamma_{0}+\gamma_{1} b\right) \ell^{*} \\
& =\left(\ell \gamma_{0}^{*}+\gamma_{0} \ell^{*}\right)-\ell \gamma_{1}^{*} b^{-1}+\gamma_{1} b \ell^{*} .
\end{aligned}
$$

The first term, $\ell \gamma_{0}^{*}+\gamma_{0} \ell^{*}$, has the form $\beta+\beta^{*}$, which is central in $\mathbb{Z C}$ [15, Theorem III.2.1]. Writing

$$
\beta=\sum_{z_{i} \in S_{1}} \beta_{i} z_{i}+\sum_{\ell_{i} \in S_{2}} \beta_{i}^{\prime} \ell_{i}
$$

where the elements in $S_{1}$ are central and those in $S_{2}$ are not, we have

$$
\beta^{*}=\sum_{z_{i} \in S_{1}} \beta_{i} z_{i}+s \sum_{\ell_{i} \in S_{2}} \beta_{i}^{\prime} \ell_{i} \quad \text { and } \quad \beta+\beta^{*}=2 \sum \beta_{i} z_{i}+(1+s) \tau, \quad \tau \in \mathbb{Z C},
$$

so $(1-s)\left(\beta+\beta^{*}\right)=2(1-s) \sum \beta_{i} z_{i}$. The product of $1-b^{2}$ with the remaining terms in $(3.2)$ is

$$
\left(1-b^{2}\right)\left(-\ell \gamma_{1}^{*} b^{-1}+\gamma_{1} b \ell^{*}\right)=\left(\gamma_{1} \ell^{*}+\ell \gamma_{1}^{*}\right) b-\ell \gamma_{1}^{*} b^{-1}-\gamma_{1} b^{3} \ell^{*} .
$$

Now $\left(\gamma_{1} \ell^{*}+\ell \gamma_{1}^{*}\right) b$ is of the form $\left(\beta+\beta^{*}\right) b$ so, as before, it is an element with even coefficients, and the same is true for $-\ell \gamma_{1}^{*} b^{-1}-\gamma_{1} b^{3} \ell^{*}=-\left(\ell \gamma_{1}^{*}+\gamma_{1} \ell^{*}\right) b^{-1}$. All this shows that $\mu \mu^{f}$ belongs to the group ring of the centre of $L$, which is an abelian group of exponent four, and that $\mu \mu^{f}$ has the form $1+\tau$, where $\tau$ has even coefficients. So $\mu \mu^{f}$ is trivial. Since it has 1 in its support, $\mu \mu^{f}=1$, so $\mu^{f}=\mu^{-1}$ and $\mu$ is $f$-unitary.

Case 2. If $\ell=g b, g \in \mathcal{C}$, then

$$
\mu \mu^{f}=-1+\left(1-b^{2}\right)(1-s)\left[g b \gamma^{f}-\gamma g^{-1} b^{-1}+4 \gamma \gamma^{f}\right] .
$$

Now

$$
\begin{aligned}
g b \gamma^{f}-\gamma g^{-1} b^{-1} & =g b\left(\gamma_{0}^{*}-\gamma_{1}^{*} b^{-1}\right)-\left(\gamma_{0}+\gamma_{1} b\right) g^{-1} b^{-1} \\
& =g b \gamma_{0}^{*}-g \gamma_{1}^{*}-\gamma_{0} g^{*} b^{-1}+\gamma_{1} g^{*}
\end{aligned}
$$

The element $\gamma_{1} g^{*}-g \gamma_{1}^{*}$, being of the form $\beta-\beta^{*}$, is a multiple of $1-s$, so the product with $1-s$ gives an element with even coefficients. The product of $g b \gamma_{0}^{*}-\gamma_{0} g^{*} b^{-1}$ with $1-b^{2}$ is $\left(g \gamma_{0}^{*}+\gamma_{0} g^{*}\right) b-\left(\gamma_{0} g *+g \gamma_{0}^{*}\right) b^{-1}$. As in Case 1, this an element in the group ring of the centre of $L$ with even coefficients and, as before, we obtain $\mu \mu^{f}=-1$. Thus $\mu^{f}=-\mu^{-1}$ and $\mu$ is $f$-unitary. 
Theorem 3.3. Suppose $L=A\langle b\rangle$ is the product of a Hamiltonian Moufang 2-loop, $A$, and a cyclic group $\langle b\rangle$ of order $2, b \notin A$. Suppose also that $x^{-1} a x=a^{-1}$ for all $a \in A$ and all $x \notin A$. Then every unit in $\mathbb{Z} L$ is $f$-unitary, where $\operatorname{ker} f=A$.

Proof. Let $\mu$ be a unit in $\mathbb{Z} L$ and write $\mu=\mu_{1}+\mu_{2} b, \mu_{1}, \mu_{2} \in \mathbb{Z} A$. As noted in the proof of Theorem 3.2, the map $f$ coincides with the canonical involution $\alpha \mapsto \alpha^{*}$ in the alternative loop ring $\mathbb{Z} A$. Thus $\mu^{f}=\mu_{1}^{*}-b \mu_{2}^{*}$. Furthermore, since $b a^{-1}=a b$ for $a \in A$, we have $b \mu_{2}^{*}=\mu_{2} b$, so

$$
\begin{aligned}
\mu \mu^{f} & =\left(\mu_{1}+\mu_{2} b\right)\left(\mu_{1}^{*}-\mu_{2} b\right) \\
& =\mu_{1} \mu_{1}^{*}-\left(\mu_{2} b\right)\left(\mu_{2} b\right)-\mu_{1}\left(\mu_{2} b\right)+\left(\mu_{2} b\right) \mu_{1}^{*} .
\end{aligned}
$$

Using diassociativity and $b \mu_{2}=\mu_{2}^{*} b$, the product $\left(\mu_{2} b\right)\left(\mu_{2} b\right)=\mu_{2} \mu_{2}^{*} b^{2}=\mu_{2} \mu_{2}^{*}$. If $x$ is in the support of $\mu_{2} b$, then $x \notin A$, so $\left(\mu_{2} b\right) \mu_{1}^{*}=\mu_{1}\left(\mu_{2} b\right)$. Thus $\mu \mu^{f}=\mu_{1} \mu_{1}^{*}-\mu_{2} \mu_{2}^{*}$ is a unit in the loop ring $\mathbb{Z} A$ and is hence trivial. For $\alpha \in \mathbb{Z} A$, let $\bar{\alpha}$ denote the image of $\alpha$ in $\mathbb{Z}\left[L / L^{\prime}\right]$. Since $\bar{\alpha}^{*}=\bar{\alpha}$, we have $\overline{\mu \mu}^{f}=\bar{\mu}_{1}^{2}-\bar{\mu}_{2}^{2}$. It follows that $\bar{\mu}_{1} \pm \bar{\mu}_{2}$ are units in the group ring of an abelian group of exponent two. By Theorem 2.2, they are both trivial. There are four cases to consider. The arguments in each case are similar. We present one.

Suppose $\bar{\mu}_{1}+\bar{\mu}_{2}=\bar{a}$ and $\bar{\mu}_{1}-\bar{\mu}_{2}=\bar{\ell}, a, \ell \in A$. It follows readily that $\bar{a}=\bar{\ell}=\bar{\mu}_{1}$ and $\bar{\mu}_{2}=\overline{0}$, so $\mu_{1}=a+(1-s) \gamma_{1}$ and $\mu_{2}=(1-s) \gamma_{2}$ with $\gamma_{1}, \gamma_{2} \in \mathbb{Z} L$. So

$$
\begin{aligned}
\mu \mu^{f} & =\left[a+(1-s) \gamma_{1}\right]\left[a^{*}+(1-s) \gamma_{1}^{*}\right]-2(1-s) \gamma_{2} \gamma_{2}^{*} \\
& =1+(1-s)\left(\gamma_{1} \ell^{*}+\ell \gamma_{1}^{*}-2 \gamma_{2} \gamma_{2}^{*}\right)
\end{aligned}
$$

(since $a^{*}=a^{-1}$ ). Now $\gamma_{1} \ell^{*}+\ell \gamma_{1}^{*}$ has the form $\beta+\beta^{*}$, so it can be written $2 \sum \beta_{i} z_{i}+$ $(1+s) \tau$, with $z_{i} \in A$ central and $\tau \in \mathbb{Z} A$. Since the product of such an element with $1-s$ has even coefficients, 1 is in the support of the trivial unit $\mu \mu^{f}$, so $\mu \mu^{f}=1, \mu^{f}=\mu^{-1}$ and $\mu$ is $f$-unitary.

Let $16 \Gamma_{2} c_{2}$ be the group $\left\langle a, b \mid a^{4}=b^{4}=\left(a^{2}, b\right)=1,(a, b)=a^{2}\right\rangle$. (The notation is due to Hall and Senior [17].) The RA loop $M\left(16 \Gamma_{2} c_{2}, *, a^{2}\right)$, which Chein denotes $M_{32}\left(16 \Gamma_{2} c_{2}, 16 \Gamma_{2} c_{2}, 16 \Gamma_{2} c_{2}^{\sharp}, 16 \Gamma_{2} c_{2}^{\sharp}\right)[\mathbf{5}]$, is $32 / 65$ in the catalogue of Moufang loops of small order by Goodaire et al. [16]. As an RA loop, this can be generated by $a, b$ and a third element $u$. The unique non-identity/commutator in this loop is $s=a^{2}=u^{2}$.

Theorem 3.4. Let $E$ be an abelian group of exponent two. Then every unit of $M\left(16 \Gamma_{2} c_{2}, *, a^{2}\right) \times E$ is $f$-unitary, where ker $f=\langle a, u\rangle \times E$.

Proof. By Lemma 3.1, we may assume that $E$ is trivial. In [11, Theorem 6.1], it is proven that the unit loop of $\mathbb{Z} L$ is $\pm L \mathcal{V}$, where $\mu \in \mathcal{V}$ has the form

$$
\mu=1+(1-s)\left(1+b^{2}\right)\left[\left(\alpha_{0}+\alpha_{1} a+\alpha_{2} b+\alpha_{3} a b\right)+\left(\beta_{0}+\beta_{1} a+\beta_{2} b+\beta_{3} a b\right) u\right],
$$

where $\alpha_{0}, \ldots, \alpha_{3}, \beta_{0}, \ldots, \beta_{3}$ are integers satisfying a certain condition that is not relevant here. It suffices to show that every unit of this form is $f$-unitary. On the one hand (by 
Theorem 6.1 of $[\mathbf{1 1}])$, we have

$$
\begin{aligned}
\mu^{-1}=1+(1-s)\left(1+b^{2}\right)\left[\left(\alpha_{0}+\alpha_{1} a^{-1}-\alpha_{2} b\right.\right. & \left.-\alpha_{3} a b\right) \\
& \left.+\beta_{0} u^{-1}+\beta_{1}(a u)^{-1}-\beta_{2} b u-\beta_{3} a b \cdot u\right] .
\end{aligned}
$$

On the other hand,

$$
\begin{aligned}
\mu^{f}=1+(1-s)\left(1+b^{2}\right)\left[\left(\alpha_{0}+\alpha_{1} a^{-1}\right.\right. & \left.-\alpha_{2} b^{-1}-\alpha_{3}(a b)^{-1}\right) \\
& \left.+\beta_{0} u^{-1}+\beta_{1}(a u)^{-1}-\beta_{2}(b u)^{-1}-\beta_{3}(a b \cdot u)^{-1}\right] .
\end{aligned}
$$

Thus it is enough to show that

$$
\begin{aligned}
(1-s)\left(1+b^{2}\right)\left[-\alpha_{2} b-\alpha_{3} a b-\right. & \beta_{2} b u-\beta_{3} a b \cdot u \\
& \left.+\alpha_{2} b^{-1}+\alpha_{3}(a b)^{-1}+\beta_{2}(b u)^{-1}+\beta_{3}(a b \cdot u)^{-1}\right]=0 .
\end{aligned}
$$

Remembering that every element $x \notin A=\langle a, u\rangle$ has square $b^{2}$, we have

$$
x^{-1}-x=x^{3}-x=x\left(x^{2}-1\right)=x\left(b^{2}-1\right) .
$$

Since $\left(1+b^{2}\right)\left(b^{2}-1\right)=0$, the result follows.

We conclude this section with a final scenario in which the units of a loop ring are $f$-unitary.

Theorem 3.5. Suppose $L$ is an $R A$ loop containing a subloop $A$ of index 2 and that $L=A \cup A b$ for some $b \notin A$. Suppose every subloop of $T$, the torsion subloop of $L$, is normal in $L$. If $T=\langle b\rangle \times C$ is a direct product with $b$ of order 8 and $C$ an abelian group of exponent dividing 4 , or $b$ of order 4 and $C$ an abelian group of exponent dividing 6 , then every unit of $\mathbb{Z} L$ is $f$-unitary, where $\operatorname{ker} f=A$.

Proof. The hypotheses imply that $\mathcal{U}(\mathbb{Z} L)=\mathcal{U}(\mathbb{Z} T) \cdot L$ [15, Proposition XII.1.3]. Suppose $b$ has order 8 and $C$ has exponent dividing 4. Applying Theorem 2 of $[\mathbf{1}]$ with $G=T$, part 5.3 says every unit of $\mathbb{Z} T$ is $f$-unitary. Since the elements of $L$ are $f$-unitary, the same holds true for $\mathcal{U}(\mathbb{Z} L)$.

Suppose $b$ has order 4 and $C$ has exponent dividing 6 . This time, part 5.2 of $[\mathbf{1}$, Theorem 2] says that every unit of $\mathbb{Z} T$ is $f$-unitary so again, the same holds true for $\mathcal{U}(\mathbb{Z} L)$.

\section{The classification}

In this section, we state and complete the proof of the major theorem of this paper, which follows.

Theorem 4.1. Let $L$ be an $R A$ loop with torsion subloop $T$. Then $\mathcal{U}_{f}(\mathbb{Z} L)=\mathcal{U}(\mathbb{Z} L)$ for some homomorphism $f: L \rightarrow\{ \pm 1\}$ if and only if $L$ is described by one of the conditions below. 
(1) Every subloop of $T$ is normal in $L$, and $T$ is either an abelian group of exponent dividing 4 or 6 or a Hamiltonian Moufang 2-loop.

(2) Every subloop of $T$ is normal in $L$ and $T=\langle b\rangle \times C$ with $b$ of order 8 and $C$ an abelian group of exponent dividing 4 or $b$ of order 4 and $C$ an abelian group of exponent dividing 6.

(3) $L=A\langle b\rangle$ is the product of a Hamiltonian Moufang 2-loop $A$ and a cyclic group $\langle b\rangle$ of order $2, b \notin A$, and $x^{-1} a x=a^{-1}$ for all $a \in A$ and all $x \notin A$.

(4) $L$ is the direct product of an abelian group of exponent two and the loop $M\left(16 \Gamma_{2} c_{2}, *, a^{2}\right)$.

(5) $L=\mathcal{C} \times\langle b\rangle \times E$ is the direct product of the Cayley loop, $\mathcal{C}$, a cyclic group $\langle b\rangle$ of order 4 , and an abelian group $E$ of exponent two.

In $\S \S 2$ and 3, we showed that loops with the indicated structure have the desired property, so here, we assume that all units of $\mathbb{Z} L$ are $f$-unitary and show that $L$ has one of the structures described. Some of our arguments follow those of Bovdi [1], whose paper inspired this one. Throughout, $L$ is an RA loop, $f: L \mapsto\{ \pm 1\}$ is a homomorphism and $\mathcal{U}_{f}(\mathbb{Z} L)=\mathcal{U}(\mathbb{Z} L)$ (whether or not this is explicitly stated).

We begin with an elementary lemma.

Lemma 4.2. If $f(\ell)=1$ for all $\ell \in L$, then $\mathcal{U}_{f}(\mathbb{Z} L)=\mathcal{U}(\mathbb{Z} L)$ implies that all units in $\mathbb{Z} L$ are trivial, so $L$ is described by Theorem 4.1 (1).

Proof. Let $\mu=\sum_{\ell \in L} \mu_{\ell} \ell$ be a unit. We have $\mu \mu^{f}= \pm 1$. Since $\mu^{f}=\sum \mu_{\ell} \ell^{-1}$, the coefficient of 1 in $\mu \mu^{f}$ is $\sum \mu_{\ell}^{2}>0$. In particular, $\mu \mu^{f}=+1$, implying that $\mu_{\ell_{0}}= \pm 1$ for a unique $\ell_{0}$ and $\mu_{\ell}=0$ for all $\ell \neq \ell_{0}$. Thus $\mathcal{U}(\mathbb{Z} L)= \pm L$ and reference to Theorem 2.3 completes the proof.

Now assume that $f$ is not identically 1 on $L$. We collect some information about this situation, which we assume for the rest of this paper.

First of all, $A=\operatorname{ker} f$ is a subloop of $L$ of index 2, hence normal, and $L=A \cup A b$ for any $b \notin A$. If $B$ is any commutative subloop of an RA loop $L$ and $x \in L$ is any element, the subloop $\langle B, x\rangle$ generated by $B$ and $x$ is a group [15, Corollary IV.2.4]. In the present context, it follows that $A$ cannot be commutative. This implies, in particular, that the unique non-identity commutator $s$ of $L$ is in $A$, so $f(s)=1$.

Next, if $b$ is not central, then $b$ cannot commute element-wise with $A$. To see why, remember that $L=A \cup A b$, so $a b=b a$ for all $a \in A$ would imply that $b$ commutes with all elements of $L$ (by diassociativity). As noted in $\S 1$, this implies that $b$ associates with all pairs of elements of $L$; in other words, $b$ would be central.

Also, since $f(a)=1$ for all $a \in A$, Lemma 4.2 says that $\mathcal{U}(\mathbb{Z} A)= \pm A$, so, by Theorem 2.3, every subloop of the torsion subloop $T(A)$ of $A$ is normal in $A$, and $T(A)$ is an abelian group of exponent dividing 4 or 6 , or a Hamiltonian Moufang 2-loop.

The next lemma allows us to assume that $L \backslash A$ contains a torsion element. 
Lemma 4.3. Let $L$ be an $R A$ loop with torsion subloop $T$. Assume that $\mathcal{U}_{f}(\mathbb{Z} L)=$ $\mathcal{U}(\mathbb{Z} L)$ for some $f: L \rightarrow\{ \pm 1\}$. Then $x^{-1} t x \in\langle t\rangle$ for any $t \in T$ and any $x \notin T$.

Proof. Let $\hat{t}=1+t+t^{2}+\cdots+t^{n-1}, n$ the order of $t$. Since $\alpha=(1-t) x \hat{t}$ is nilpotent, $\mu=1+\alpha$ is a unit with inverse $\mu^{-1}=1-\alpha$. By assumption, $\mu^{f}= \pm \mu^{-1}$.

If $\mu^{f}=\mu^{-1}$, then $1+\alpha^{f}=1-\alpha$, so $\alpha+\alpha^{f}=0$. This implies that

$$
(1-t) x \hat{t} \pm \hat{t}^{f} x^{-1}\left(1 \pm t^{-1}\right)=0,
$$

so $t x=t^{i} x^{-1}$ for some $i$, or $t x=t^{i} x^{-1} t^{-1}$ for some $i$. In the first case, $x^{2} \in\langle t\rangle$ has finite order, which is not true. The second case implies that for some $j, x^{-1}=t^{j} x t=t^{j+1} x$ or $s t^{j+1} x$, either possibility again contradicting the fact that $x^{2}$ has infinite order.

If $\mu^{f}=-\mu^{-1}$, then $1+\alpha^{f}=-1+\alpha$, so $\alpha-\alpha^{f}=2$. Since $t \alpha=0$, we obtain $2 t=-t \alpha^{f}$, hence $2=-\alpha^{f}$, which is not true ( $\alpha^{f}$ is a zero divisor). So $\mu^{f}=-\mu^{-1}$ cannot occur and the proof is complete.

Corollary 4.4. Let $L$ be an $R A$ loop with torsion subloop $T$. If every element of $L \backslash A$ has infinite order and all units of $\mathbb{Z} L$ are $f$-unitary for some $f$, then $L$ has the property described in Theorem $4.1(1)$.

Proof. The hypothesis says that the torsion subloop $T$ of $L$ is the torsion subloop of $A$. Thus, as observed in remarks just preceding Lemma 4.3, $T$ is an abelian group of exponent dividing 4 or 6 of a Hamiltonian Moufang 2-loop and every subloop of $T$ is normal in $A$. Because of the lemma, every subloop of $T$ is actually normal in $L$. The result follows.

In view of Corollary 4.4, we may assume in the sequel that $L \backslash A$ contains an element $b$ of finite order. Since the torsion subloop of $A$ has exponent dividing 4 or 6 , if $b \in L \backslash A$ has finite order, $b^{2} \in A$ has order $2,3,4$ or 6 . If $b^{2}$ has order $3, b^{3}$ has order 2 . Note that $b^{3} \notin A$ since $b^{2} \in A$ and $b \notin A$. If $b^{2}$ has order 6 , then $b^{3}$ has order 4 and, again, $b^{3} \notin A$. Thus it suffices in the sequel to assume that $L \backslash A$ contains an element $b$ of order dividing 8 .

Throughout this section, we use the notation $T(A)$ and $T(L)$ to denote the torsion subloops of $A$ and $L$, respectively. It is easy to see that $T(L)=T(A) \cup T(A) b$.

Case 1: $\boldsymbol{L} \backslash \boldsymbol{A}$ contains an element $\boldsymbol{b}$ of order 2. If $b$ is central, then $L=A \times\langle b\rangle$ and, since all units of $\mathbb{Z} A$ are trivial, the same holds for $\mathbb{Z} L$ by [15, Theorem VIII.3.1, Step 1]. Theorem 2.3 then says that $L$ meets the criteria of Theorem 4.1 (1).

Suppose $b$ is not central. As noted in the remarks after Lemma 4.2, this implies $a b \neq$ $b a$ for some $a \in A$. Since $(1-b) a(1+b)$ has square $0, \mu=1+(1-b) a(1+b)$ is a unit with $\mu^{-1}=1-(1-b) a(1+b)$ and $\mu^{f}=1+\left(1-b^{-1}\right) a^{-1}\left(1+b^{-1}\right)$ (recall that $\alpha \rightarrow \alpha^{f}$ is an antiautomorphism). By hypothesis, $\mu^{f}= \pm \mu^{-1}$ and since $\mu^{-1}$ and $\mu^{f}$ each have augmentation 1 , necessarily, $\mu^{f}=+\mu^{-1}$. This implies $\left(1-b^{-1}\right) a^{-1}\left(1+b^{-1}\right)=$ $-(1-b) a(1+b)$, hence $a^{-1}+a^{-1} b^{-1}-b^{-1} a^{-1}-b^{-1} a^{-1} b^{-1}=-a-a b+b a+b a b$, so

$$
a^{-1}+a^{-1} b+a+a b=b a+b a b+b^{-1} a^{-1}+b^{-1} a^{-1} b^{-1} .
$$


Since $a^{-1}$ is in the support of the left-hand side, it is in the support of the right-hand side as well, that is,

$$
a^{-1} \in\left\{b a, b a b, b^{-1} a^{-1}, b^{-1} a^{-1} b^{-1}\right\} .
$$

If $a^{-1}=b a$, then $b=a^{-2}$ is central [15, Theorem IV.1.8], which is not true. If $a^{-1}=$ $b^{-1} a^{-1}$, then $b=1$, which is not true, and if $a^{-1}=b^{-1} a^{-1} b^{-1}=b a^{-1} b=s a^{-1} b^{2}=$ $s a^{-1}$, then $s=1$, which is not true. The only possibility is $a^{-1}=b a b$, which implies $a^{-1} b^{-1}=b a=s a b$, so $a^{-1}=s a$ and $a^{2}=s$.

Now fix an $a_{0} \in A$ which does not commute with $b$ (hence $a_{0}^{2}=s$ ) and let $a \in A$. If $a b \neq b a$, then $a^{2}=s$. Suppose $a b=b a$. Then $a a_{0}$ does not commute with $b$, so

$$
\left(a a_{0}\right)^{2}=s= \begin{cases}a^{2} a_{0}^{2}=s a^{2} & \text { if } a a_{0}=a_{0} a, \\ s a^{2} a_{0}^{2}=a^{2} & \text { if } a a_{0}=s a_{0} a .\end{cases}
$$

We claim that the second case cannot occur. To see why, suppose the contrary (and remember that $a b=b a$ and $\left.b^{2}=1\right)$. Let $a_{1}=a a_{0}$. Then $a_{1}^{2}=s$, so $\left(a_{1} b\right)^{2}=s a_{1}^{2} b^{2}=1$. Consider

$$
\begin{aligned}
\left(a_{1}+a_{1} b\right)^{2} & =a_{1}^{2}+a_{1}^{2} b+a_{1} b a_{1}+\left(a_{1} b\right)^{2} \\
& =s+s b+s a_{1}^{2} b+1=s+s b+b+1=(1+s)(1+b) .
\end{aligned}
$$

Thus $\left(a_{1}+a_{1} b\right)(1-s)$ has square 0 , so $\mu=1+\left(a_{1}+a_{1} b\right)(1-s)$ is a unit with $\mu^{-1}=$ $1-\left(a_{1}+a_{1} b\right)(1-s)$. Remembering that $s \in A$ (so $f(s)=1$ ), we have

$$
\begin{aligned}
\mu^{f} & =1+\left(a_{1}^{f}+\left(a_{1} b\right)^{f}\right)(1-s) \\
& =1+\left(a_{1}^{-1}-\left(a_{1} b\right)^{-1}\right)(1-s) \\
& =1+\left(s a_{1}-b^{-1} a_{1}^{-1}\right)(1-s) \\
& =1+\left(s a_{1}-s b a_{1}\right)(1-s) \\
& =1+\left(s a_{1}+a_{1} b\right)(1-s) .
\end{aligned}
$$

Because $\epsilon\left(\mu^{-1}\right)=\epsilon\left(\mu^{f}\right)=+1$, we must have $\mu^{f}=\mu^{-1}$, so $1+\left(s a_{1}+a_{1} b\right)(1-s)=$ $1-\left[\left(a_{1}+a_{1} b\right)(1-s)\right]$, implying

$$
\begin{aligned}
\left(s a_{1}+a_{1} b+a_{1}+a_{1} b\right)(1-s) & =0, \\
\left(2 a_{1} b+a_{1}+s a_{1}\right)(1-s) & =0, \\
2 a_{1} b+a_{1}+s a_{1}-2 s a_{1} b-s a_{1}-a_{1} & =0,
\end{aligned}
$$

the last equation giving $2 a_{1} b(1-s)=0$, which is not true. This verifies the claim and allows us to conclude that

$$
a^{2}= \begin{cases}s & \text { if } a b \neq b a \\ 1 & \text { if } a b=b a\end{cases}
$$

This implies that $A$ has exponent four (so $L$ is a torsion loop) and also that $b^{-1} a b=a^{-1}$ for all $a \in A$, because

$$
b^{-1} a b= \begin{cases}a=a^{-1} & \text { if } a b=b a, \\ s a=a^{-1} & \text { if } a b \neq b a .\end{cases}
$$


Now let $b_{1} \in L$ be any element not in $A$. Then $b_{1}=a_{1} b$ for some $a_{1} \in A$. If $a_{1} b \neq b a_{1}$, then $b_{1}^{2}=s a_{1}^{2} b^{2}=s a_{1}^{2}=1$, so if $b_{1}$ is central (for any such $b_{1}$ ), then $L$ is as described in Theorem 4.1 (1), from what we have already seen. On the other hand, if $b_{1}$ is never central, then, again using what we have already shown, $b_{1}^{-1} a b_{1}=a^{-1}$ and $L$ is as described by Theorem $4.1(3)$.

Suppose $a_{1} b=b a_{1}$ (and hence $\left(a_{1}, b, x\right)=1$ for any $x \in L$ ). By the known structure of $A$, every subloop of $A$ is normal in $A$. Since also $b_{1}^{-1} a b_{1}=a$ or $a^{-1}$, every subloop of $A$ is normal in $L$. If $x \notin A$, write $x=a_{2} b_{1}$ for some $a_{2} \in A$. Then

$$
b_{1}^{-1} x b_{1}=b_{1}^{-1}\left(a_{2} b_{1}\right) b_{1}=b_{1}^{-1} a_{2}=b_{1} a_{2}= \begin{cases}a_{2} b_{1} & \text { if } a_{2} b_{1}=b_{1} a_{2}, \\ s a_{2} b_{1} & \text { if } a_{2} b_{1} \neq b_{1} a_{2} .\end{cases}
$$

In the latter case, $b^{-1} x b_{1}=a_{2}^{3} b_{1}=\left(a_{2} b_{1}\right)^{3}$ because $a_{2}^{2}=s$. It follows that every subloop of the torsion loop $L$ is normal in $L$ and $L$ is as described in Theorem 4.1 (1). (See remarks preceding Lemma 4.3.) This concludes Case 1 and permits us to assume, henceforth, that $L \backslash A$ does not contain an element of order 2 .

Case 2: $L \backslash A$ contains an element $b$ of order 4 . We analyse two subcases.

Case 2(a): $\langle\boldsymbol{b}\rangle$ is normal in $\boldsymbol{L}$. First suppose that $a b=b a$ for all $a \in T(A)$. If $T(A)$ is an Abelian group of exponent dividing 4, then so is $T(L)=T(A) \cup T(A) b$. It is easy to see that $x^{-1} t x=t$ for every $t \in T(L)$. Together with Lemma 4.3, this implies that every subloop of $T(L)$ is normal in $L$, so the structure of $L$ is given by (1) of Theorem 4.1. If $T(A)$ has exponent dividing 6 but not 4, then, applying Theorem 1 of $[\mathbf{1}]$ to $G=T(L)$ with Bovdi's $A$ our $T(A)$ there appear to be two possibilities (which we label as in [1]):

1. $b a b^{-1}=a^{-1}$ for all $a \in T(A)$; or

5.2. $T(L)$ is the direct product $\langle b\rangle \times C$ with $b$ of order 4 and $C$ an Abelian group of exponent dividing 6 .

Since we are assuming that $b$ commutes with all elements of $T(A)$ and since $T(A)$ contains an element of order 3 , statement 1 cannot occur, so it is 5.2 that holds and the structure of $L$ is described by (3) of Theorem 4.1.

Suppose, on the other hand, that $T(A)$ is a Hamiltonian 2-loop. Thus $T(A)=K \times E$, where $E$ is an elementary abelian 2-group and $K=Q_{8}$, the quaternion group of order 8, or $K=\mathcal{C}$, the Cayley loop [15, $\S$ II.4]. If $b^{2} \in K$, then as a central element of order 2, $b^{2}=s$. Take an $a \in K$ with $a^{2}=s$. Then $(a b)^{2}=a^{2} b^{2}=1$. This contradicts the explicit assumption made after Case 1 that $L \backslash A$ contains no elements of order 2 . Thus $b^{2} \notin K$, so $b^{2}=k e, k \in K, 1 \neq e \in E$. Write $E=E_{1} \times\langle e\rangle$. Then $\left[K \times E_{1}\right] \cap\langle b\rangle=\{1\}$ since $b^{2}=k_{1} e_{1}, k_{1} \in K, e_{1} \in E_{1}$, implies $k_{1} e_{1}=k e \in K \times E$, so $e=e_{1} \in E_{1}$, a contradiction. Also $K \times E_{1} \unlhd T(L)$ because every subloop of $T(A)$ is normal in $A$ and $b$ commutes with every element of $T(A)$. Thus $T(L)=T(A)\langle b\rangle=K \times E_{1} \times\langle b\rangle$.

Choose $a, c \in K$ such that $\langle a, c\rangle \cong Q_{8}$. Note that $b c=c b$ because $c \in T(A)$. Suppose $x \in L$ has infinite order. Then $x^{2} \in A$ has infinite order, so $x^{2} c$ has infinite order. By Lemma 4.3, $\left(x^{2} c\right)^{-1}(a b)\left(x^{2} c\right) \in\langle a b\rangle$, contradicting $\left(x^{2} c\right)^{-1}(a b)\left(x^{2} c\right)=c^{-1}(a b) c$ (by 
centrality of $\left.x^{2}\right)=c^{-1} a c b=a^{-1} b$. Thus no such $x$ exists, so $L=T(L)$. In particular, $K$ cannot be associative, so $L$ is as described by Theorem $4.1(5)$.

We may now assume that there exists $a \in T(A)$ with $a b \neq b a$. Thus $a^{-1} b a=s b=b^{3}$, so $b^{2}=s$. Also $(a b)^{2}=s a^{2} b^{2}=a^{2}$. Since we are assuming that $L \backslash A$ contains no elements of order 2 , we have $a^{2} \neq 1$. Elements of odd order in an RA loop are central, so the order of $a$ is not 3 , nor is it 6 since, if this were the case, $b^{-1} a b=a^{5}=s a$ would imply $a^{4}=s$ and $a^{8}=1$. The only possibility is that $a$ has order 4 . Form the unit $\mu=1+(1-a) b \hat{a}$, $\hat{a}=1+a+a^{2}+a^{3}$, and note that $\mu^{-1}=1-(1-a) b \hat{a}$ and $\mu^{f}=1-\hat{a}^{f} b^{-1}\left(1-a^{3}\right)$. We have $\mu^{f}= \pm \mu^{-1}$ and, since $\epsilon\left(\mu^{f}\right)=1=\epsilon\left(\mu^{-1}\right)$, it must be that $\mu^{f}=+\mu^{-1}$. Since $\hat{a}^{f}=\hat{a}$, this implies

$$
\begin{gathered}
\left(1+a+a^{2}+a^{3}\right) b^{-1}\left(1-a^{3}\right)=(1-a) b\left(1+a+a^{2}+a^{3}\right), \\
b^{-1}+a b^{-1}+a^{2} b^{-1}+a^{3} b^{-1}+a b+a b a+a b a^{2}+a b a^{3} \\
=b+b a+b a^{2}+b a^{3}+b^{-1} a^{3}+a b^{-1} a^{3}+a^{2} b^{-1} a^{3}+a^{3} b^{-1} a^{3}
\end{gathered}
$$

and so

$$
b \in\left\{b^{-1}, a b^{-1}, a^{2} b^{-1}, a^{3} b^{-1}, a b, a b a, a b a^{2}, a b a^{3}\right\} .
$$

If $b=b^{-1}$, then $b^{2}=1$; if $b=a b^{-1}$, then $a=b^{2}$ commutes with $b$; if $b=a^{3} b^{-1}$, then $b^{2}=a^{3}$ and $a$ is central; if $b=a b$, then $a=1$; if $b=a b a^{2}=s b a^{3}$, then $a^{3}=s$ and $a$ is central; if $b=a b a^{3}=s b a^{4}=s b$, then $s=1$. None of these conclusions is correct, so $b=a^{2} b^{-1}$ or $b=a b a=s b a^{2}$, both of which give $a^{2}=b^{2}=s$. Now fix an $a_{0} \in T(A)$ with $a_{0} b \neq b a_{0}\left(\right.$ so $\left.a_{0}^{2}=s\right)$. Let $a \in T(A)$ and suppose $a b=b a$. Then $a a_{0}$ does not commute with $b$, so

$$
s=\left(a_{0} a\right)^{2}= \begin{cases}a_{0}^{2} a^{2}=s a^{2} & \text { if } a_{0} a=a a_{0}, \\ s a_{0}^{2} a^{2}=a^{2} & \text { if } a_{0} a \neq a a_{0},\end{cases}
$$

and it follows that $a^{2}=1$ or $a^{2}=s$. If $a^{2}=s$, however, then $(a b)^{2}=1$, contradicting our assumption that $L \backslash A$ contains no elements of order 2. So we have

$$
a b \neq b a \Longleftrightarrow a^{2}=s \quad \text { and } \quad a b=b a \Longleftrightarrow a^{2}=1 .
$$

In particular, this implies that $\left(a_{1} b\right)^{2}=b^{2}=s$ for any $a_{1} \in T(A)$ since $\left(a_{1} b\right)^{2}=a_{1}^{2} b^{2}$ or $s a_{1}^{2} b^{2}$ according to whether $a_{1} b=b a_{1}$ or $a b \neq b a$, respectively. So $a_{1} b$ has order 4 and $\left\langle a_{1} b\right\rangle \unlhd L$ because the subloop in question contains $s$ [15, Corollary IV.1.11].

Let $a, a_{1} \in T(A)$. If $x=a_{1}$, we have $x^{-1} a x \in\langle a\rangle$ because every subloop of $T(A)$ is normal in $A$. If $x=a_{1} b, a_{1} \in T(A)$, applying to $x$ what we have learned about $b$, then

$$
x^{-1} a x= \begin{cases}a=a^{-1} & \text { if } a x=x a, \\ s a=a^{3} & \text { if } a x \neq x a .\end{cases}
$$

Also, for any $x \in L$ and any $a_{1} \in T(A), x^{-1}\left(a_{1} b\right) x=a_{1} b$ or $x^{-1}\left(a_{1} b\right) x=s\left(a_{1} b\right)=\left(a_{1} b\right)^{3}$. We have shown that every subloop of $T(L)$ is normal in $L$. Since $T(L)=T(A) \cup T(A) b$ is not an abelian group, $T(L)$ is Hamiltonian. It is a 2-loop because $T(A)$ is and $(a b)^{2}=s$ for all $a \in T(A)$. So $L$ is described by Theorem $4.1(1)$. 
Case 2(b): $\langle\boldsymbol{b}\rangle$ is not normal in $\boldsymbol{L}$. In particular, this means that $b$ is not central and $s \notin\langle b\rangle[\mathbf{1 5}$, Corollary IV.1.11]. As noted in the remarks after Lemma 4.2, there exists $a \in A$ with $a b \neq b a$. The element $\mu=1+(1-b) a \hat{b}, \hat{b}=1+b+b^{2}+b^{3}$, is a unit with $\mu^{-1}=1-(1-b) a \hat{b}$. Since $\hat{b}^{f}=1-b^{-1}+b^{-2}-b^{-3}=1-b+b^{2}-b^{3}$, we have $\mu^{f}=1+\left(1-b+b^{2}-b^{3}\right) a^{-1}\left(1+b^{-1}\right)$. Since $\mu^{-1}$ and $\mu^{f}$ have augmentation 1 , we have $\mu^{f}=+\mu^{-1}$, so

$$
\left(1-b+b^{2}-b^{3}\right) a^{-1}\left(1+b^{-1}\right)=-\left[(1-b) a\left(1+b+b^{2}+b^{3}\right)\right],
$$

which implies

$$
\begin{aligned}
a^{-1}+a^{-1} b^{-1} & +b^{2} a^{-1}+b^{2} a^{-1} b^{-1}+a+a b+a b^{2}+a b^{3} \\
& =b a+b a b+b a b^{2}+b a b^{3}+b a^{-1}+b a^{-1} b^{-1}+b^{3} a^{-1}+b^{3} a^{-1} b^{-1}
\end{aligned}
$$

hence

$$
a \in\left\{b a, b a b, b a b^{2}, b a b^{3}, b a^{-1}, b a^{-1} b^{-1}, b^{3} a^{-1}, b^{3} a^{-1} b^{-1}\right\} .
$$

If $a=b a$, then $b=1$; if $a=b a b=s a b^{2}$, then $b^{2}=s$; if $a=b a b^{2}=b^{3} a$, then $b^{3}=1$; if $a=b a b^{3}=s a b^{4}=s a$, then $s=1$; if $a=b a^{-1}$, then $b=a^{2}$ is central; and if $a=b^{3} a^{-1}$, then $b^{3}=a^{2}$ is central. None of the conclusions here is correct, so either $a=b a^{-1} b^{-1}=s a^{-1}$ and $a^{2}=s$, or $a=b^{3} a^{-1} b^{-1}=s b^{3} b^{-1} a^{-1}=s b^{2} a^{-1}$ and $a^{2}=s b^{2}$. We claim that the latter cannot occur, that is $a^{2} \neq s b^{2}$.

To see why, suppose $a^{2}=s b^{2}$ and note then that $(a+b)^{2}=a^{2}+b^{2}+a b+b a=$ $(1+s)\left(a b+b^{2}\right)$. This would mean that $1+(a+b)(1-s)$ is a unit with $\mu^{-1}=1-(a+b)(1-s)$ and $\mu^{f}=1+(1-s)\left(a^{-1}-b^{-1}\right)$. Since $\mu^{f}=\mu^{-1}$ (in view of augmentations), we would have

$$
(1-s)\left(a^{-1}-b^{-1}\right)=-[(a+b)(1-s)],
$$

and so

$$
a^{-1}-b^{-1}-s a^{-1}+s b^{-1}=-a-b+s a+s b,
$$

implying $a \in\left\{s a, s b, b^{-1}, s a^{-1}\right\}$. Now $a \neq s a$ because $s \neq 1 ; a \neq s b$ because $a$ and $b$ do not commute; $a \neq b^{-1}$ because $b \notin A$. If $a=s a^{-1}$, then $a^{2}=s$ and $b^{2}=s a^{2}=1$, which is not true. This justifies our claim and establishes that if $a b \neq b a$, then $a^{2}=s$.

Now fix $a_{0} \in A$ with $a_{0} b \neq b a_{0}$ (thus $a_{0}^{2}=s$ ) and let $a \in A$ be arbitrary. If $a b \neq b a$, then $a^{2}=s$. If $a b=b a$, then $a a_{0}$ does not commute with $b$, so

$$
\left(a a_{0}\right)^{2}=s= \begin{cases}a^{2} a_{0}^{2}=s a^{2} & \text { if } a a_{0}=a_{0} a \\ s a^{2} a_{0}^{2}=a^{2} & \text { if } a a_{0}=s a_{0} a\end{cases}
$$

Thus $a^{2}=1$ or $a^{2}=s$ according to whether $a a_{0}=a_{0} a$ or $a a_{0} \neq a_{0} a$, respectively. In particular, we learn that $A=T(A)$ is a torsion loop of exponent four. Since $A$ is never commutative, $A$ must be a Hamiltonian Moufang 2-loop.

Let $e \in A$ and $e^{2}=1$. Then $e$ is central in $L$ because it is central in $A$ and commutes with $b$. (We know that if $e b \neq b e$, then $e^{2}=s$.) Moreover, $e b$ is not central. If $a \in A$ has order 4 , then $a^{2}=s$. If $a b$ were central, then $a b$ would have order $4-(a b)^{2}=a^{2} b^{2} \neq 1$ 
(since $b^{2} \neq s$ ) - and we would be finished by Case 2(a). So we may assume that $a b$ is not central for any $a \in A$. Finally, if $a \in A$ and $a$ has order 4 , then $a^{2}=s$ and $a a_{0} \neq a_{0} a$, so $a$ is not central. It follows that $\mathcal{Z}(L)$, the centre of $L$, is $\left\{e \in A \mid e^{2}=1\right\}$. Furthermore, if $a$ has order 4 , none of $a, b, a b$ is central, so the LC property implies $a b \neq b a$. This establishes that

$$
a b \neq b a \Longleftrightarrow a \text { has order } 4 \text {. }
$$

We claim that $x^{-1} a x=a^{-1}$ for any $a \in A$ and any $x \in L$. This is true if $a$ has order 2, since then $a$ is central and $x^{-1} a x=a=a^{-1}$. If $a$ has order 4 , then $a b \neq b a, a^{2}=s$ and $b^{-1} a b=s a=a^{3}=a^{-1}$. Finally, consider $\left(a_{1} b\right)^{-1} a\left(a_{1} b\right)$ for $a_{1} \in A$. If $a_{1} b=b a_{1}$, then $a_{1}$ has order 2 because of (4.2), so $a_{1}$ is central and $\left(a_{1} b\right)^{-1} a\left(a_{1} b\right)=b^{-1} a b=a^{-1}$. If $a_{1} b \neq b a_{1}$, then (4.2) implies $\left(a_{1} b\right)^{2}=s a_{1}^{2} b^{2}=b^{2}$. Having established Case 2(a), we may assume that $a_{1} b$ is not central. Also, since $b^{2} \neq s,\left(a_{1} b\right)^{2} \neq s$, so $s \notin\left\langle a_{1} b\right\rangle$. This implies that $\left\langle a_{1} b\right\rangle$ is not normal in $L$ and, replacing $b$ by $a_{1} b$ in this case, we obtain $\left(a_{1} b\right)^{-1} a\left(a_{1} b\right)=a^{-1}$.

Suppose there exists $w \in \mathcal{Z}(L) \backslash A$. Let $a \in A$ have order 4 . Then $w^{-1} a w=a^{-1}=a$, a contradiction. Thus $\mathcal{Z}(L) \subseteq A$. If there exist $x, y, u \in A$ which do not associate, then $L=\langle x, y, u, \mathcal{Z}(L)\rangle \subseteq A$, which is false. It follows that $A$ is a group, so $A=$ $Q_{8} \times E$, where $E$ is an elementary abelian 2-group. Since $A$ is a group, the identity $(x y, z, w)=(x, z, w)(y, z, w)$, which holds in any RA loop [15, Theorem IV.1.14], and the fact that $L=A \cup A b$ show that there exist $x, y \in A$ with $(x, y, b) \neq 1$. Thus $L=M\left(Q_{8} \times E, *, b^{2}\right)$. Since $\langle b\rangle$ is not normal, $b^{2} \notin\{1, s\}$, so $b^{2} \notin Q_{8}$ and we may write $b^{2}=q e, q \in Q_{8}, 1 \neq e \in E$. Since $E=E_{0} \times\langle e\rangle$ for some subgroup $E_{0}$ of $E$, we have $L=M\left(Q_{8} \times\langle e\rangle \times E_{0}, *, b^{2}\right)=M\left(Q_{8} \times\langle e\rangle, *, b^{2}\right) \times E_{0}$ by [15, Proposition V.1.6]. As the only RA loop of order 32 with exactly three squares, the RA loop $M\left(Q_{8} \times\langle e\rangle, *, b^{2}\right)$ has to be the loop denoted $32 / 65$ in [16]. Thus $L$ is described by Theorem 4.1 (3).

Case 3: $\boldsymbol{b}$ has order 8 . In this case, $T(A)$ has to be an abelian group since $b^{2} \in A$ is a central element of order 4 (and there are no such elements in a Hamiltonian Moufang 2-loop).

Case 3(a): $\langle\boldsymbol{b}\rangle$ is normal in $\boldsymbol{L}$. Suppose $a b=b a$ for all $a \in T(A)$. It is easy to prove that $x^{-1} t x=t$ for all $x, t \in T(L)=T(A) \cup T(A) b$ so, together with Lemma 4.3, we see that every subloop of $T(L)$ is normal in $L$. Also, $T(L)=T(A) \cup T(A) b$ is a torsion abelian group and all units in its integral group ring are $f$-unitary. Applying Theorem 1 of [1] to the group $G=T(L)$ with Bovdi's $A$ our $T(A)$, there are two possibilities (which we label as in Bovdi's Theorem):

(4) the torsion subgroup of $A /\left\langle b^{4}\right\rangle$ has exponent two and $b \bar{a} b^{-1}=\bar{a}$ for all $\bar{a} \in A /\left\langle b^{4}\right\rangle$; or

(5.3) the torsion subgroup of $T(L)$ (which is $T(L)$ itself) is the direct product of $\langle b\rangle$ and an abelian group whose order divides 4 .

Since we are assuming that $b$ commutes with all elements of $a$, Case 4 implies that $A$ is torsion, so $L=A \cup A b$ is torsion. But $L=T(L)$ contradicts the fact that $L$ is a not an abelian group. Thus we are in Case 5.3, which is described by Theorem 4.1 (2). 
Now assume that there exists $a \in T(A)$ with $a b \neq b a$. Now $a^{-1} b a \in\left\{b^{3}, b^{5}, b^{7}\right\}$ and, easily, $a^{-1} b a=b^{5}=s b$, so $b^{4}=s$. Form the unit $\mu=1+(1-a) b \hat{a}, \hat{a}=1+a+a^{2}+$ $\cdots+a^{n-1}, n$ the order of $a$. Then $\mu^{-1}=1-(1-a) b \hat{a}, \mu^{f}=1-\hat{a} b^{-1}\left(1-a^{-1}\right)$ (since $\left.\hat{a}^{f}=\hat{a}\right)$, so $\mu^{-1}=\mu^{f}$ implies

$$
(1-a) b \hat{a}=\hat{a} b^{-1}\left(1-a^{-1}\right) .
$$

Since elements of odd order are central and $T(A)$ has exponent dividing 4 or 6 , we must have $n=2, n=4$ or $n=6$. If $n=2$, Equation (4.3) is $(1-a) b(1+a)=(1+a) b^{-1}(1-a)$, so

$$
b \in\left\{a b, a b a, b^{-1}, a b^{-1}\right\} .
$$

The only possibility is $b=a b a=s a^{2} b$, giving $a^{2}=s$.

If $n=4$, we obtain

$$
b \in\left\{b^{-1}, a b^{-1}, a^{2} b^{-1}, a^{3} b^{-1}, a b, a b a, a b a^{2}, a b a^{3}\right\}
$$

and hence $a^{2}=b^{2}$ or $a^{2}=s$ (see Case 2(a)). Since $a^{2}=b^{2}$ implies that $a$ has order 8 , which is not true, we again have $a^{2}=s$.

If $n=6$, Equation (4.3) implies that

$$
b \in\left\{a b, a b a, a b a^{2}, a b a^{3}, a b a^{4}, a b a^{5}, b^{-1}, a b^{-1}, a^{2} b^{-1}, a^{3} b^{-1}, a^{4} b^{-1}, a^{5} b^{-1}\right\} .
$$

We claim that $b=a b a$, in which case $b=s b a^{2}$ and $a^{2}=s$. Indeed, there is no other possibility: $b=a b$ implies $a=1 ; b=a b a^{2}=a^{3} b$ implies $a^{3}=1$, so $a$ is central; $b=a b a^{3}=s b a^{4}$ implies $a^{4}=s$, so $a$ has order $8 ; b=a b a^{4}=a^{5} b$ implies $a^{5}=1$; $b=a b a^{5}=s b a^{6}$ implies $a^{6}=s$, so $a$ has order $12 ; b=b^{-1}$ implies $b^{2}=1 ; b=a b^{-1}$ implies $a=b^{2}$ is central; $b=a^{2} b^{-1}$ implies $a^{2}=b^{2}$, so $a$ has order $8 ; b=a^{3} b^{-1}$ implies $a^{3}=b^{2}$ is central, so $a$ is central; $b=a^{4} b^{-1}$ implies $a^{4}=b^{2}$, so $a$ has order 16 ; and $b=a^{5} b^{-1}$ implies $a^{5}=b^{2}$ is central, so $a$ is central.

These arguments show that if $a \in T(A)$ and $a b \neq b a$, then $a^{2}=s$, so $a$ has order $n=4$. Fix such an $a_{0} \in T(A)$ (thus $a_{0}^{2}=s$ ), let $a \in T(A)$ and suppose that $a b=b a$. Then $b$ and $a a_{0}$ do not commute, so $\left(a a_{0}\right)^{2}=s$. Since $T(A)$ is abelian, $s=a^{2} a_{0}^{2}=s a^{2}$, so $a^{2}=1$. This shows that $T(A)$ in fact has exponent dividing 4 and, more precisely, that if $a \in T(A)$,

$$
a^{2}=1 \Longleftrightarrow a b=b a \quad \text { and } \quad a^{2}=s \Longleftrightarrow a b \neq b a .
$$

We claim that every subloop of $T(L)=T(A) \cup T(A) b$ is normal in $L$, so that $L$ is described by Theorem $4.1(1)$.

For this, let $a, x \in T(A)$ and observe that $x^{-1} a x=a$ because $T(A)$ is abelian while $(x b)^{-1} a(x b)=b^{-1} x^{-1} a x b=b^{-1} a b=a$ or $s a$ and, in the latter case, $a b \neq b a$, so $s a=$ $a^{2} a=a^{3} \in\langle a\rangle$. Also, since $(a b)^{2}=a^{2} b^{2}$ or $s a^{2} b^{2}$ according to whether $a b=b a$ or $a b \neq b a$, respectively, observation (4.4) implies that $(a b)^{2}=b^{2}$ in any case, so $(a b)^{4}=b^{4}=s$, implying $s \in\langle a b\rangle$ and hence $\langle a b\rangle \unlhd L$. 
Case 3(b): $\langle\boldsymbol{b}\rangle$ is not normal in $\boldsymbol{L}$. Recall that this condition implies that $s \notin\langle b\rangle$. Moreover, $b$ cannot be central so, as noted earlier, there exists $a \in A$ with $a b \neq b a$. Form the unit $\mu=1+(1-b) a \hat{b}$. Then $\mu$ is a unit with inverse $\mu^{-1}=1-(1-b) a \hat{b}$ and $\mu^{f}=1+\hat{b}^{f} a^{-1}\left(1+b^{-1}\right)$. Since $\hat{b}^{f}=1-b+b^{2}-b^{3}+b^{4}-b^{5}+b^{6}-b^{7}, \epsilon\left(\mu^{f}\right)=1$, so we must have $\mu^{f}=\mu^{-1}$. This implies that

$$
\hat{b}^{f} a^{-1}\left(1+b^{-1}\right)=-(1-b) a \hat{b}
$$

A calculation which, by now, should be familiar, shows that

$$
\begin{array}{r}
b \in\left\{b a, b a b, b a b^{2}, b a b^{3}, b a b^{4}, b a b^{5}, b a b^{6}, b a b^{7}, b a^{-1}, b a^{-1} b^{-1},\right. \\
\left.b^{3} a^{-1}, b^{3} a^{-1} b^{-1}, b^{5} a^{-1}, b^{5} a^{-1} b^{-1}, b^{7} a^{-1}, b^{7} a^{-1} b^{-1}\right\} .
\end{array}
$$

We show that $a=b a^{-1} b^{-1}$ or $a=b^{3} a^{-1} b^{-1}$ or $a=b^{5} a^{-1} b^{-1}$ or $a=b^{7} a^{-1} b^{-1}$ by eliminating all other possibilities.

If $a=b a$, then $b=1$; if $a=b a b=s a b^{2}$, then $b^{2}=s$ and, similarly, each of the conditions $a=b a b^{2}, a=b a b^{3}, a=b a b^{4}, a=b a b^{5}, a=b a b^{6}$ and $a=b a b^{7}$ implies that $s \in\langle b\rangle$, which is not correct. If $a=b a^{-1}$, then $b=a^{2}$ is central and hence commutes with $b$; similarly, each of $a=b^{3} a^{-1}, a=b^{5} a^{-1}$ and $a=b^{7} a^{-1}$ implies that $b$ is central, which is not correct. Thus it is indeed the case that

$$
a=b a^{-1} b^{-1}=s a^{-1} \quad \text { so that } a^{2}=s,
$$

or

$$
a=b^{3} a^{-1} b^{-1}=s b^{2} a^{-1} \quad \text { so that } a^{2}=s b^{2},
$$

or

$$
a=b^{5} a^{-1} b^{-1}=s b^{4} a^{-1} \quad \text { so that } a^{2}=s b^{4},
$$

or

$$
a=b^{7} a^{-1} b^{-1}=s b^{6} a^{-1} \quad \text { so that } a^{2}=s b^{6} .
$$

In every case, $a^{8}=1$. Now fix $a_{0} \in A$ with $a_{0} b \neq b a_{0}$ (so that $a_{0}^{8}=1$ ) and take $a \in A$. If $a b \neq b a$, then $a^{8}=1$ as above. If $a b=b a$, then $a a_{0}$ does not commute with $b$, so $\left(a a_{0}\right)^{8}=1$. Now

$$
\left(a a_{0}\right)^{2}= \begin{cases}a^{2} a_{0}^{2} & \text { if } a a_{0}=a_{0} a, \\ s a^{2} a_{0}^{2} & \text { if } a a_{0} \neq a_{0} a,\end{cases}
$$

so, in any event, $\left(a a_{0}\right)^{8}=a^{8} a_{0}^{8}=a^{8}=1$. It follows that $A$ has exponent dividing 8 , so $A=T(A)$ is abelian, which is not the case (see remarks after Lemma 4.2).

Acknowledgements. E.G.G. is again grateful to FAPESP of Brazil and to the Instituto de Matemática e Estatística of the Universidade de São Paulo, where he is always made to feel very welcome and where the environment for pursuing mathematics is marvellous.

This research was supported by a Discovery Grant from the Natural Sciences and Engineering Research Council of Canada and by FAPESP, Proc. 2000/07290-0 and CNPq., Proc. 300243/79-0 (RN) of Brazil. 


\section{References}

1. A. A. Bovd, Unitarity of the multiplicative group of an integral group ring, Mat. USSR Sb. 47(2) (1984), 377-389.

2. A. A. Bovdi AND S. K. Sehgal, Unitary subgroup of integral group rings, Manuscr. Math. 76 (1992), 213-222.

3. A. Bovdi AND L. ERDEI, Unitary units in modular group algebras of 2-groups, Commun. Alg. 28(2) (2000), 625-630.

4. V. Bovdi, L. G. Kovács And S. K. Sehgal, Symmetric units in modular group algebras, Commun. Alg. 24(3) (1996), 803-808.

5. O. Chein, Moufang loops of small order, Memoirs of the American Mathematical Society, vol. 13 (1978).

6. O. Chein and E. G. Goodaire, Loops whose loop rings are alternative, Commun. Alg. 14(2) (1986), 293-310.

7. O. Chein and E. G. Goodaire, Loops whose loop rings in characteristic 2 are alternative, Commun. Alg. 18(3) (1990), 659-688.

8. A. Giambruno and C. Polcino Milies, Unitary units and skew elements in group algebras, Manuscr. Math. 111 (2003), 195-209.

9. J. Z. GonçAlves And D. S. PAssman, Unitary units in group algebras, Israel J. Math. 125 (2001), 131-155.

10. E. G. Goodaire, Alternative loop rings, Publ. Math. Debrecen 30 (1983), 31-38.

11. E. G. Goodaire, Six Moufang loops of units, Can. J. Math. 44(5) (1992), 951-973.

12. E. G. Goodaire And M. M. Parmenter, Units in alternative loop rings, Israel J. Math. $\mathbf{5 3}(2)(1986), 209-216$.

13. E. G. Goodaire And C. Polcino Milies, On the loop of units of an alternative loop ring, Nova J. Alg. Geom. 3(3) (1995), 199-208.

14. E. G. Goodaire And C. Polcino Milies, Central idempotents in alternative loop algebras, Nova J. Math. Game Theory Alg. 5(3) (1996), 207-214.

15. E. G. Goodaire, E. Jespers And C. Polcino Milies, Alternative loop rings, NorthHolland Mathematical Studies, vol. 184 (Elsevier, 1996).

16. E. G. Goodaire, S. MAY And M. Raman, The Moufang loops of order less than 64 (Nova Science, Commack, NY, 1999).

17. M. HALl JR AND J. K. SEnIOR, The groups of order $2^{n}$ (Macmillan, London, 1964).

18. G. Higman, The units of group rings, Proc. Lond. Math. Soc. 46 (1940), 231-248.

19. Y. LI, Units of $\mathbb{Z}\left[G \times C_{2}\right]$, Quest. Math. 21 (1998), 201-218. 